VOL. $70(2004) \quad[257-266]$

\title{
NON-MINIMAL TREE ACTIONS AND THE EXISTENCE OF NON-UNIFORM TREE LATTICES
}

\author{
Lisa Carbone
}

\begin{abstract}
A uniform tree is a tree that covers a finite connected graph. Let $X$ be any locally finite tree. Then $G=\operatorname{Aut}(X)$ is a locally compact group. We show that if $X$ is uniform, and if the restriction of $G$ to the unique minimal $G$-invariant subtree $X_{0} \subseteq X$ is not discrete then $G$ contains non-uniform lattices; that is, discrete subgroups $\Gamma$ for which $\Gamma \backslash G$ is not compact, yet carries a finite $G$-invariant measure. This proves a conjecture of Bass and Lubotzky for the existence of nonuniform lattices on uniform trees.
\end{abstract}

\section{INTRODUCTION}

Let $X$ be a locally finite tree and let $G=\operatorname{Aut}(X)$. Then $G$ is naturally a locally compact group $([3,4])$. For a discrete subgroup $\Gamma \leqslant G$, the vertex stabilizers $\Gamma_{x}$, $x \in V X$, are finite groups $[3]$. Let $V(\Gamma \backslash X)$ be the vertex set of the quotient graph $\Gamma \backslash X$. As in [3] and [4] we call $\Gamma$ an $X$-lattice, or a tree lattice if

$$
\operatorname{Vol}(\Gamma \backslash \backslash X)=\sum_{x \in V(\Gamma \backslash X)} \frac{1}{\left|\Gamma_{x}\right|}
$$

is finite, and a uniform $X$-lattice if $\Gamma \backslash X$ is a finite graph, non-uniform otherwise.

Following [3] we call $X$ uniform if $X$ is the universal cover of a finite connected graph. We call $X$ rigid if $G=\operatorname{Aut}(X)$ is discrete, and $X$ is minimal if $G$ acts minimally on $X$, that is, there is no proper $G$-invariant subtree [4]. If $X$ is uniform then there is always a unique minimal $G$-invariant subtree $X_{0} \subseteq X([4,(5.7),(5.11)$, (9.7)]). We call $X$ virtually rigid if $X_{0}$ is rigid.

The following results of Bass and Tits [5] and Bass and Lubotzky [4] indicate that uniform trees with discrete groups of automorphisms cannot give rise to non-uniform lattices.

The author was supported in part by NSF grant \#DMS-9800604.

Copyright Clearance Centre, Inc. Serial-fee code: 0004-9727/04 SA2.00+0.00. 
Proposition $0.1 .([5,(5.5)]$.$) Let X$ be a locally finite tree. If $X$ is uniform and rigid then all $X$-lattices are uniform.

PRoposition 0.2 . ([4, (3.7)].) Let $X$ be a locally finite tree. If $X$ is uniform and virtually rigid then all $X$-lattices are uniform.

In analogy with Borel's classical theorem establishing the co-existence of uniform and non-uniform lattices in connected non-compact semisimple Lie groups, Bass and Lubotzky conjectured that under some natural assumptions $G=\operatorname{Aut}(X)$ contains both uniform and non-uniform lattices ([4, Chapter 7,8$])$. In particular, they conjectured that when $G$ contains uniform lattices, the only obstruction to the existence of non-uniform lattices is virtual rigidity of $X([4$, Chapter 7,8$])$. Here we present a proof of this conjecture. We use a theorem of Bass and Kulkarni [3] which states that $G=$ Aut $(X)$ contains a uniform lattice if and only if $X$ is uniform. Our main theorem is the following.

THEOREM 0.3. If $X$ is uniform and not virtually rigid then $G$ contains a nonuniform $X$-lattice.

In [6], the author proved Theorem 0.3 for minimal actions assuming also the (necessary) Bass-Tits criterion for non-discreteness of $G([5,(5.5)])$, which is equivalent to non-rigidity of $X$. That is, in [6] the author proved:

Theorem 0.4. ([6].) Let $X$ be a uniform tree, and let $G=\operatorname{Aut}(X)$. If $G$ is not discrete and acts minimally on $X$, then there is a non-uniform $X$-lattice $\Gamma \leqslant G$.

Here we no longer assume that $G$ acts minimally. Suppose that $X$ is a uniform tree and let $X_{0} \subseteq X$ be the unique minimal $G$-invariant subtree of $X$, also a uniform tree. Let $G_{0}=\left.\operatorname{Aut}(X)\right|_{X_{0}}$. In $([8])$ we showed that $G_{0}=\operatorname{Aut}\left(X_{0}\right)$. If $X$ is not virtually rigid, that is $X_{0}$ is not rigid, then by Theorem $0.4 G_{0}$ contains a non-uniform $X_{0}$-lattice $\Gamma_{0}$. Thus our task is to show that $\Gamma_{0}$ extends to a non-uniform $X$-lattice $\Gamma \leqslant G=\operatorname{Aut}(X)$. This is achieved by Theorems 3.1 and 3.4 in Section 3 .

Theorems 0.3 and 0.4 together give a complete proof of the Bass-Lubotzky conjecture for the existence of non-uniform lattices on uniform trees $([4$, Chapter 7,8$])$. Together with [2], and with [9] and [10] where we address the Bass-Lubotzky existence question in the case that $X$ is not uniform, we have answered the Bass-Lubotzky conjectures in full. We refer the reader to [7] for an overview of the Bass-Lubotzky conjectures and their proofs.

\section{Tree lattices, edge-indexed graphs, volumes and Coverings}

Let $\Gamma$ be a group acting without inversions on a tree $X$. The fundamental theorem of Bass and Serre $([\mathbf{1}, \mathbf{1 2}])$ states that $\Gamma$ is encoded (up to isomorphism) in a 'quotient graph of groups' $\mathbb{A}=\Gamma \backslash \backslash X([\mathbf{1}, \mathbf{1 2}])$. Conversely a graph of groups $\mathbb{A}$ gives rise to a 
group $\Gamma=\pi_{1}(\mathbf{A}, a), a \in V \mathbb{A}$, acting on a tree $X=\widetilde{(\mathbb{A}, a)}$ without inversions, and the vertex stabilizers $\Gamma_{x}, x \in V X$, are (conjugate to) the vertex groups of $\mathbb{A}([1,12])$.

Now assume that $X$ is locally finite, and that $\Gamma$ acts on $X$ with quotient graph of groups $\mathbb{A}=\Gamma \backslash \backslash X$. Then $\mathbb{A}$ naturally gives rise to an 'edge-indexed' graph $(A, i)$, defined as follows. The graph $A$ is the underlying graph of $\mathbf{A}$ with vertex set $V A$, edge set $E A$, initial and terminal functions $\partial_{0}, \partial_{1}: E A \longmapsto V A$ which pick out the endpoints of an edge and with fixed point free involution - : $E A \longmapsto E A$ which reverses the orientation. The indexing $i: E A \longmapsto \mathbb{Z}_{>0}$ of $(A, i)$ is defined to be the group theoretic index

$$
i(e)=\left[\mathcal{A}_{\partial_{0} e}: \alpha_{e}\left(\mathcal{A}_{e}\right)\right]
$$

where

$$
\left(\mathcal{A}_{a}\right)_{a \in V A} \text { and }\left(\mathcal{A}_{e}=\mathcal{A}_{\bar{e}}\right)_{e \in E A}
$$

are the vertex and edge groups of $\mathbb{A}$, and $\alpha_{e}: \mathcal{A}_{e} \hookrightarrow \mathcal{A}_{\partial_{0} e}$ are the boundary monomorphisms of $\mathbb{A}$. We write $(A, i)=I(\mathbb{A})$ when $i(e)=\left[\mathcal{A}_{\partial_{0} e}: \alpha_{e}\left(\mathcal{A}_{e}\right)\right]$ for data

$$
\left\{\mathcal{A}_{a}, \mathcal{A}_{e}=\mathcal{A}_{\bar{e}}, \alpha_{e}: \mathcal{A}_{e} \hookrightarrow \mathcal{A}_{\partial_{0} e}\right\}
$$

from $\mathbb{A}$. Conversely, an edge-indexed graph $(A, i)$ is defined to be a graph $A$ and an assignment $i: E A \longmapsto \mathbb{Z}_{>0}$ of a positive integer to each oriented edge. Then $(A, i)$ determines a universal covering tree $X=\widetilde{(A, i)}$ up to isomorphism $([\mathbf{3}, \mathbf{4}])$, and every edge-indexed graph arises from a tree action [4]. Here we assume $i(e)$ is finite for each $e \in E A$. Under this assumption the universal covering tree $X=\widetilde{(A, i)}$ is locally finite $([3,4])$.

Given an edge-indexed graph $(A, i)$, a graph of groups $\mathbb{A}$ such that $I(\mathbb{A})=(A, i)$, is called a grouping of $(A, i)$. We call $\mathbb{A}$ a finite grouping if the vertex groups $\mathcal{A}_{a}$ are finite and a faithful grouping if $\mathbb{A}$ is a faithful graph of groups, that is if $\pi_{1}(\mathbb{A}, a)$, $a \in V A$ acts faithfully on $X=\widetilde{(\mathbf{A}, a)}$ [3]. If $\mathbf{A}$ is not faithful, then a faithful quotient of $\mathbf{A}$ always exists $([\mathbf{1}])$.

Lemma 1.1. $([\mathbf{3}, \mathbf{4}]$.) Let $(A, i)$ be an edge-indexed graph and let $\mathbb{A}$ be a finite faithful grouping of $(A, i)$. Then for $a \in V A, \Gamma=\pi_{1}(\mathbf{A}, a)$ is a discrete subgroup of $G=\operatorname{Aut}(X)$, where $X=\widetilde{(A, i)}$.

For an edge $e$ in $(A, i)$, define:

$$
\Delta(e)=\frac{i(\bar{e})}{i(e)} .
$$

If $\gamma=\left(e_{1}, \ldots, e_{n}\right)$ is a path, set $\Delta(\gamma)=\Delta\left(e_{1}\right) \ldots \Delta\left(e_{n}\right)$. An indexed graph $(A, i)$ is then called unimodular if $\Delta(\gamma)=1$ for all closed paths $\gamma$ in $A$. This is equivalent to unimodularity of $G=\operatorname{Aut}(X)$ where $X=\widetilde{(A, i)}[3]$. 
Assume now that $(A, i)$ is unimodular. Pick a base point $a_{0} \in V A$, and define, for $a \in V A$,

$$
N_{a_{0}}(a)=\frac{\Delta a}{\Delta a_{0}} \quad\left(=\Delta(\gamma) \text { for any path } \gamma \text { from } a_{0} \text { to } a\right) \in \mathbb{Q}>0 .
$$

For $e \in E A$, set

$$
N_{a_{0}}(e)=\frac{N_{a_{0}}\left(\partial_{0}(e)\right)}{i(e)} .
$$

Following $([4,(2.6)])$, we say that $(A, i)$ has bounded denominators if $\left\{N_{a_{0}}(e) \mid e \in E A\right\}$ has bounded denominators, that is, if for some integer $D>0, D \cdot N_{a_{0}}$ takes only integer values on edges. This condition is automatic if $A$ is finite, and since

$$
N_{a_{1}}=\frac{\Delta a_{0}}{\Delta a_{1}} N_{a_{0}}
$$

this condition is independent of $a_{0} \in V A$. As in [3] the functions $N: A \longrightarrow \mathbb{Q}_{>0}^{\times}$as above are called vertex orderings of $(A, i)$. We call $N$ integral if for all $e \in E A$, we have $N\left(\partial_{0}(e)\right) / i(e) \in \mathbb{Z}$ and hence $N(a) \in \mathbb{Z}$ for $a \in V A$.

THEOREM 1.2. ([3, (2.4)].) The following conditions on an edge-indexed graph $(A, i)$ are equivalent.

(a) $(A, i)$ admits a finite (faithful) grouping.

(b) $(A, i)$ is unimodular and has bounded denominators.

(c) $(A, i)$ admits an integral vertex ordering.

We define the volume of an indexed graph $(A, i)$ at a basepoint $a_{0} \in V A$ :

$$
\operatorname{Vol}_{a_{0}}(A, i)=\sum_{a \in V A} \frac{1}{\left((\Delta a) /\left(\Delta a_{0}\right)\right)}=\sum_{a \in V A}\left(\frac{\Delta a_{0}}{\Delta a}\right) .
$$

Then

$$
\operatorname{Vol}_{a_{1}}(A, i)=\frac{\Delta a_{0}}{\Delta a_{1}} \operatorname{Vol}_{a_{0}}(A, i),
$$

as in $\left(\left[4\right.\right.$, Chapter 2]). We write $\operatorname{Vol}(A, i)<\infty$ if $\operatorname{Vol}_{a}(A, i)<\infty$ for some, and hence every $a \in V A$.

If $\mathbb{A}$ is a finite grouping of $(A, i)$, then we have $([4,(2.6 .15)])$ :

$$
\operatorname{Vol}(\mathbb{A})=\frac{1}{\left|\mathcal{A}_{a}\right|} \operatorname{Vol}_{a}(A, i)
$$

which is automatically finite if $\operatorname{Vol}(A, i)<\infty$. 
We now describe a method for constructing $X$-lattices which follows naturally from the fundamental theory of Bass and Serre, and was first suggested in [3]. We begin with an edge-indexed graph $(A, i)$. Then $(A, i)$ determines its universal covering tree $X=\widetilde{(A, i)}$ up to isomorphism ([4, Chapter 2]). If $(A, i)$ is unimodular and has bounded denominators, then by Theorem 1.2 we can find a finite (faithful) grouping $\mathbb{A}$ of $(A, i)$. By Lemma (1.1), $\Gamma=\pi_{1}\left(\mathbb{A}, a_{0}\right), a_{0} \in V A$, is a discrete subgroup of $G=\operatorname{Aut}(X)$. If further $(A, i)$ has finite volume, then $\mathbb{A} \cong \Gamma \backslash \backslash X$ has finite volume $\operatorname{Vol}(\mathbb{A})=\operatorname{Vol}(\Gamma \backslash \backslash X)$. It follows that $\Gamma=\pi_{1}\left(\mathbb{A}, a_{0}\right)$ is an $X$-lattice, uniform if $A=\Gamma \backslash X$ is a finite graph, non-uniform otherwise.

A covering $p:(B, j) \longrightarrow(A, i)$ of edge-indexed graphs $([4,(2.5)])$, is a graph morphism $p: B \longrightarrow A$ such that for all $e \in E A, \partial_{0}(e)=a$, and $b \in p^{-1}(a)$, we have

$$
i(e)=\sum_{f \in p_{(b)}^{-1}(e)} j(f)
$$

where $p_{(b)}: E_{0}^{B}(b) \longrightarrow E_{0}^{A}(a)$ is the local map on the star $E_{0}(v)$ of a vertex $v$, that is, the set of edges with initial vertex $v$. If $b \in V B, p(b)=a \in V A$, then we can identify

$$
(\widetilde{A, i, a})=X=(\widetilde{B, j, b})
$$

so that the diagram of natural projections

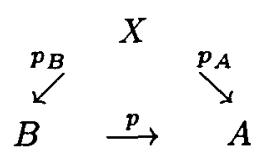

commutes. Let $G_{(B, j)}=\left\{g \in G \mid g \circ p_{B}=p_{B}\right\}$ and $G_{(A, i)}=\left\{g \in G \mid g \circ p_{A}=p_{A}\right\}$ be the groups of deck transformations of $(B, j)$ and $(A, i)$ respectively. If $p:(B, j)$ $\longrightarrow(A, i)$ is a covering of edge-indexed graphs, then we have $G_{(B, j)} \leqslant G_{(A, i)}$ ([4, Chapter 2]). If $\mathbb{A}$ is a grouping of $(A, i)$ and $\mathbb{B}$ is a grouping of $(B, j)$ then by $([4$, Chapter 2]) we have

$$
\pi_{1}(\mathbb{A}, a) \leqslant G_{(A, i)} \text { and } \pi_{1}(\mathbb{B}, b) \leqslant G_{(B, j)}
$$

\section{EXISTENCE AND STRUCTURE OF UNIQUE MINIMAL SUBTREE AND ITS QUOTIENT}

Let $X$ be a locally finite tree, and let $G=\operatorname{Aut}(X)$. We recall that $X$ is minimal if there is no proper $G$-invariant subtree. The following gives an existence theorem for minimal invariant subtrees of $X$. 
Proposition 2.1. ([4, (5.5), (5.11), (9.7)].) Let $X$ be a tree and let $G=\operatorname{Aut}(X)$. If $X$ is a uniform tree then there is a unique minimal $G$-invariant subtree $X_{G} \leqslant X$. Moreover the (hyperbolic) length function $l(G) \neq 0$, and if $\Gamma$ is any $X$-lattice, $l(\Gamma) \neq 0$ and $X_{G}=X_{\Gamma}$.

In this section we describe minimality of a group action $H \leqslant G=\operatorname{Aut}(X)$ in terms of its edge-indexed quotient graph, $(A, i)=I(H \backslash \backslash X)$, as in [4] and [8].

Let $(A, i)$ be any edge-indexed graph. We say that $(A, i)$ is minimal if $(A, i)$ is the edge-indexed quotient of a minimal tree action. A vertex $a \in V A$ is called a terminal vertex of $(A, i)$ if $\operatorname{deg}_{(A, i)}(a)=1$, where

$$
\operatorname{deg}_{(A, i)}(a)=\sum_{e \in E_{0}(a)} i(e)
$$

and $E_{0}(a)=\left\{e \in E A \mid \partial_{0} e=a\right\}$. A terminal vertex in $(A, i)$ is then a geometrically terminal vertex in the graph $A$, that is, there is a unique edge $e$ with $\partial_{0} e=a$. The following gives a geometric characterisation of a minimal edge-indexed graph.

PROPOSITION 2.2. ([8]) Let $\Gamma$ be a group acting without inversions on a tree $X$ with quotient graph of groups $\mathbb{A}=\Gamma \backslash \backslash X$ and edge-indexed quotient graph $(A, i)$ $=I(\mathbb{A})$.

(1) If $(A, i)$ is minimal then $(A, i)$ has no terminal vertices.

(2) If $(A, i)$ is finite and has no terminal vertices then $(A, i)$ is minimal.

Let $(T, i)$ be an edge-indexed graph. As in $([4,11])$ say that $(T, i)$ is a dominant rooted edge-indexed tree if $T$ is a tree and there is a vertex $a \in V T$ such that for all $e \in E T$

$$
d\left(\partial_{0} e, a\right)>d\left(\partial_{1} e, a\right) \Longrightarrow i(e)=1 .
$$

We call such a vertex $a \in V T$ a dominant root of $(T, i)$ and we write $(T, i, a)$ when $(T, i)$ is a dominant edge-indexed tree rooted at $a \in V T$.

THEOREM 2.3. ([8]) Let $(A, i)$ be a finite edge-indexed graph. Then

(1) $(A, i)$ contains a unique minimal connected subgraph $\left(A_{0}, i_{0}\right)$.

(2) $(A, i)$ has the form

$$
(A, i)=\left(A_{0}, i_{0}\right) \amalg \coprod_{a_{j} \in \Delta}\left(T_{j}, i_{j}, a_{j}\right),
$$

where $\left(T_{j}, i_{j}, a_{j}\right)$ are finite dominant-rooted edge-indexed trees with root vertices $a_{j} \in \Delta, j=1 \ldots n, \Delta \subseteq V A_{0}$ and $\left(A_{0}, i_{0}\right)$ has no terminal vertices.

Note that for $(A, i)$ as in Theorem 2.3 the covering tree $X=\widetilde{(A, i)}$ has the form

$$
X=X_{0} \amalg \coprod_{x_{j, k} \in p^{-1}\left(a_{j}\right), a_{j} \in \Delta \subseteq V A_{0}}\left(T_{j, k}, \widetilde{i_{j, k}, x_{j, k}}\right),
$$


where $j=1 \ldots n, k \geqslant 1, X_{0}=\left(\widetilde{A_{0}, i_{0}}\right), p$ is the covering map and $p\left(\widetilde{T_{j, k}}\right)=T_{j}([8])$.

\section{Existence of Non-UNiform LatTices}

Let $X$ be a locally finite tree. Let $H \leqslant G=\operatorname{Aut}(X)$ and let $G_{H}=\{g \in G \mid$ $p \circ g=p\}$ be the deck transformation group of $H$, where $p: X \longrightarrow H \backslash X$ is the quotient morphism. Let $(A, i)=I(H \backslash \backslash X)$. Then $G_{(A, i)}=G_{H}$. Let $\left(A_{0}, i_{0}\right)$ be the unique minimal subgraph of $(A, i)$ as in Theorem 2.3. Let $X_{0} \subseteq X$ be the unique minimal subtree of $X$. Then by $[8], X_{0}=\left(\widetilde{A_{0}, i_{0}}\right)$ and $H$ acts minimally on $X_{0}$. Our main theorem is the following.

THEOREM 3.1. Let $X$ be a uniform tree and let $H \leqslant G=\operatorname{Aut}(X)$. Let $X_{0} \subseteq X$ be the unique minimal $G$-invariant subtree of $X$. Let $G_{0}=\left.\operatorname{Aut}(X)\right|_{X_{0}}$ $=\operatorname{Aut}\left(X_{0}\right),(A, i)=I(H \backslash \backslash X)$, and let $\left(A_{0}, i_{0}\right)$ be the unique minimal subgraph of $(A, i)$. Assume that $G_{0}$ is not discrete ( $X_{0}$ is not rigid). Then

(i) There is a non-uniform $X_{0}$-lattice $\Gamma_{0} \leqslant G_{\left(A_{0}, i_{0}\right)} \leqslant G_{0}$.

(ii) $\Gamma_{0}$ extends to a non-uniform $X$-lattice $\Gamma \leqslant G_{(A, i)} \leqslant G=\operatorname{Aut}(X)$.

The author proved (i) of Theorem 3.1 in [6] where the assumptions on $X$ and $G$ were restated as combinatorial conditions on $(A, i)$. It remains to prove (ii). We shall give a constructive proof of (ii) by constructing the appropriate (infinite) edge-indexed graph $(B, j)$ and taking a finite faithful grouping $\mathbb{B}$ of $(B, j)$ of finite volume so that $\pi_{1}(\mathbb{B}, b)$ is a lattice, for $b \in V B$. In order to do this we describe the combinatorial restatement of the assumptions of Theorem 3.1 used in [6].

By [3] we have the following equivalent conditions:

(1) $X$ is a uniform tree.

(2) There is a uniform $X$-lattice $\Lambda \leqslant G_{H}=G_{(A, i)}$.

(3) $(A, i)$ is unimodular and finite.

(4) $\bar{H}$ is unimodular and $H \backslash X$ is finite, where $\bar{H}$ denotes the closure of $H$.

Similarly we have the following equivalent conditions:

(1) $X_{0} \subseteq X$ is uniform.

(2) 0 There is a uniform $X_{0}$-lattice $\Lambda_{0} \leqslant G_{\left(A_{0}, i_{0}\right)}$.

$(3)_{0} \quad\left(A_{0}, i_{0}\right)$ is unimodular and finite.

The assumption that $X_{0}$ is not rigid ( $G_{0}$ is not discrete) is equivalent (by [5]) to the assumption that $\left(A_{0}, i_{0}\right)$ is 'non-discretely ramified'. As in $([4,5])$ we say that an edge-indexed graph $(A, i)$ is non-discretely ramified if:

there exists $e \in E A$ such that $i(e) \geqslant 3$, or $i(e)=2$ and $e$ is not separating, or $i(e)=2$, and $\left(A_{1}(e), i\right)$ is either a ramified tree, or an unramified graph, 
where

$$
\left(A_{1}(e), i\right)=\left\{v \in V A \mid d\left(v, \partial_{1}(e)\right)>d\left(v, \partial_{0}(e)\right)\right\}
$$

If $(A, i)$ is minimal this simplifies to:

there exists $e \in E A$ such that $i(e) \geqslant 3$, or $i(e)=2$ and $\partial_{0} e$ is not a geometrically terminal vertex.

Let $(A, i)$ be a finite edge-indexed graph. We say that $(A, i)$ is virtually discretely ramified if the unique minimal subgraph $\left(A_{0}, i_{0}\right)$ is discretely ramified. We can now describe our combinatorial restatement of (i) of Theorem 3.1 proven in [6].

Theorem 3.2. ([6]) Let $X_{0}$ be a uniform tree, let $H_{0} \leqslant G_{0}=\operatorname{Aut}\left(X_{0}\right)$ and let $\left(A_{0}, i_{0}\right)=I\left(H_{0} \backslash \backslash X_{0}\right)$. If $H_{0}$ acts minimally on $X_{0}$ and is not discrete $\left(X_{0}\right.$ is not rigid) then there is a non-uniform $X_{0}$-lattice $\Gamma_{0} \leqslant G_{\left(A_{0}, i_{0}\right)} \leqslant G_{0}$. Equivalently, assume that $\left(A_{0}, i_{0}\right)$ is finite, unimodular, minimal and non-discretely ramified. Then $\left(A_{0}, i_{0}\right)$ has a covering $p_{0}:\left(B_{0}, j_{0}\right) \longrightarrow\left(A_{0}, i_{0}\right)$ such that $\left(B_{0}, j_{0}\right)$ is infinite, unimodular, has finite volume and bounded denominators.

If instead $X_{0}$ is the unique minimal invariant subtree of a uniform tree $X$, we obtain:

Corollary 3.3. Let $X$ be a uniform tree and let $H \leqslant G=\operatorname{Aut}(X)$. Let $X_{0} \subseteq X$ be the unique minimal $G$-invariant subtree of $X$, also a uniform tree. Let $G_{0}=\left.\operatorname{Aut}(X)\right|_{X_{0}}=\operatorname{Aut}\left(X_{0}\right),(A, i)=I(H \backslash \backslash X)$, and let $\left(A_{0}, i_{0}\right)$ be the unique minimal subgraph of $(A, i)$. If $X_{0}$ is not rigid then there is a non-uniform $X_{0}$-lattice $\Gamma_{0} \leqslant G_{\left(A_{0}, i_{0}\right)} \leqslant G_{0}$. Equivalently, assume that $\left(A_{0}, i_{0}\right)$ is finite, unimodular, minimal and non-discretely ramified. Then $\left(A_{0}, i_{0}\right)$ has a covering $p_{0}:\left(B_{0}, j_{0}\right) \longrightarrow\left(A_{0}, i_{0}\right)$ such that $\left(B_{0}, j_{0}\right)$ is infinite, unimodular, has finite volume and bounded denominators.

It remains to show that $\Gamma_{0}$ extends to a non-uniform $X$-lattice $\Gamma \leqslant G_{(A, i)} \leqslant G$. We achieve this with the following theorem. Our strategy is to start with a minimal edge-indexed graph $\left(B_{0}, j_{0}\right)$ that admits a non-uniform lattice, and extend this to a non-minimal edge-indexed graph $(B, j)$ that also admits a non-uniform lattice.

THEOREM 3.4. Let $\left(A_{0}, i_{0}\right)$ be an edge-indexed graph that is finite, unimodular, minimal and non-discretely ramified. Let $p_{0}:\left(B_{0}, j_{0}\right) \rightarrow\left(A_{0}, i_{0}\right)$ be a covering such that $\left(B_{0}, j_{0}\right)$ is infinite, unimodular, has finite volume and bounded denominators. Let $(A, i)$ be obtained from $\left(A_{0}, i_{0}\right)$ by attaching to vertices $a_{k} \in \Delta, k=1 \ldots n$, $\Delta \subseteq V A_{0}$ finite dominant-rooted edge-indexed trees $\left(T_{k}, i_{k}, a_{k}\right), k=1 \ldots n$. Let $(B, j)$ be obtained from $\left(B_{0}, j_{0}\right)$ by attaching to each $b_{k}^{t} \in p_{0}^{-1}\left(a_{k}\right)$ a copy of $\left(T_{k}, i_{k}, a_{k}\right)$, $k=1, \ldots, n, t>0$, denoted $\left(\widetilde{T_{k}, i_{k}, a_{k}}\right)$. Then there is a covering $p:(B, j) \longrightarrow(A, i)$ such that $(B, j)$ is infinite, unimodular, has finite volume and bounded denominators. 
PROOF: The existence of a covering $p_{0}:\left(B_{0}, j_{0}\right) \longrightarrow\left(A_{0}, i_{0}\right)$ is guaranteed by Theorem 3.2 , and $p_{0}$ extends to $p:(B, j) \rightarrow(A, i)$ in such a way that

$$
\left.p\right|_{\left(B_{0}, j_{0}\right)}=p_{0} \quad \text { and } \quad p\left(\widehat{T_{k}, i_{k}, a_{k}}\right)=\left(T_{k}, i_{k}, a_{k}\right)
$$

Moreover $(B, j)$ is automatically infinite. Since we are attaching finite trees $\left(T_{k}, i_{k}, a_{k}\right)$, $k=1, \ldots, n$, to $\left(A_{0}, i_{0}\right)$ at single vertices, $(A, i)$ is unimodular, and since $\left(B_{0}, j_{0}\right)$ is unimodular, it follows that $(B, j)$ is unimodular. Let

$$
V_{k}=\operatorname{Vol}_{v_{k}}\left(T_{k}, i_{k}, a_{k}\right), \quad k=1, \ldots, n \text {. }
$$

Let $V=\max \left\{V_{1}, V_{2}, \ldots, V_{n}\right\}$. Choose $b_{0} \in V B_{0}$ and let $V_{0}=\operatorname{Vol}_{b_{0}}\left(B_{0}, j_{0}\right)$. Then

$$
\begin{aligned}
\operatorname{Vol}_{b_{0}}(B, j) & =\sum_{v \in V B} \frac{1}{\left(\Delta v / \Delta b_{0}\right)} \\
& =\sum_{v \in p^{-1}\left(v_{1}\right)} \frac{V_{1}}{\left(\Delta v / \Delta b_{0}\right)}+\sum_{v \in p^{-1}\left(v_{2}\right)} \frac{V_{2}}{\left(\Delta v / \Delta b_{0}\right)}+\cdots+\sum_{v \in p^{-1}\left(v_{n}\right)} \frac{V_{n}}{\left(\Delta v / \Delta b_{0}\right)} \\
& \leqslant \sum_{v \in p^{-1}\left(v_{1}\right)} \frac{V}{\left(\Delta v / \Delta b_{0}\right)}+\sum_{v \in p^{-1}\left(v_{2}\right)} \frac{V}{\left(\Delta v / \Delta b_{0}\right)}+\cdots+\sum_{v \in p^{-1}\left(v_{n}\right)} \frac{V}{\left(\Delta v / \Delta b_{0}\right)} \\
& =V \sum_{v \in V B_{0}} \frac{1}{\left(\Delta v / \Delta b_{0}\right)} \\
& =V V_{0} \\
& <\infty
\end{aligned}
$$

Hence $(B, j)$ has finite volume. Let $b_{0} \in V B_{0}$. Then

$$
\left\{\frac{\Delta x}{\Delta b_{0}} \mid x \in V B_{0}\right\} \subset \mathbb{Q}
$$

has bounded denominators, since $\left(B_{0}, j_{0}\right)$ has bounded denominators. Consider

$$
\left\{\frac{\Delta y}{\Delta b_{0}} \mid y \in p_{B}^{-1}\left(T_{k}\right)\right\}=\left\{\frac{\Delta y}{\Delta v_{k}} \frac{\Delta v_{k}}{\Delta b_{0}} \mid y \in p_{B}^{-1}\left(T_{k}\right)\right\}
$$

Then the denominator of $\left(\Delta v_{k}\right) /\left(\Delta b_{0}\right)$ is bounded, since $v_{k} \in V B_{0}$, and the denominator of $(\Delta y) /\left(\Delta v_{k}\right)$ can increase only by a bounded amount for $y \in p_{B}^{-1}\left(T_{k}\right)$, since $T_{k}$ is finite for each $k=1, \ldots, n$. It follows that $(B, j)$ has bounded denominators. $\square$

COROLlaRY 3.5. In the setting of Theorem 3.4, there is a non-uniform lattice $\Gamma \leqslant G_{(B, j)} \leqslant G_{(A, i)}$.

Proof: Since $(B, j)$ is unimodular and has bounded denominators, by Theorem $1.2(B, j)$ admits a finite faithful grouping $\mathbb{B}$. Let $b \in V B, p(b)=a \in V A$, and set

$$
\Gamma=\pi_{1}(\mathbb{B}, b) \text { and } X=(\widetilde{A, i, a})=(\widehat{B, j, b}) \text {. }
$$


Then $\Gamma \leqslant G_{(B, j)} \leqslant G_{(A, i)} \leqslant G=$ Aut $(X)$. By Lemma (1.1), $\Gamma$ is a discrete subgroup of $G$. Since $\operatorname{Vol}(B, j)<\infty$,

$$
\operatorname{Vol}(\mathbb{B})=\operatorname{Vol}(\Gamma \backslash \backslash X)<\infty .
$$

Thus $\Gamma$ is an $X$-lattice, non-uniform since $(B, j)$ is infinite.

The subgroup $\Gamma \leqslant G$ is the non-uniform lattice, conjectured to exist in ([4, Chapter $7,8])$ and our proofs of Theorems 0.3 and 3.1 are complete.

\section{REFERENCES}

[1] H. Bass, 'Covering theory for graphs of groups', J. Pure Appl. Algebra 89 (1993), 3-47.

[2] H. Bass, L. Carbone and G. Rosenberg, 'The existence theorem for tree lattices', in Progress in Mathematics 176 (Birkhauser, Boston, 2000), pp. 167-184.

[3] H. Bass and R. Kulkarni, 'Uniform tree lattices', J. Amer. Math. Soc. 3 (1990), 843-902.

[4] H. Bass and A. Lubotzky, Tree lattices, Progress in Mathematics 176 (Birkhauser, Boston, 2000).

[5] H. Bass and J. Tits, A discreteness criterion for certain tree automorphism groups, Progress in Mathematics 176 (Birkhauser, Boston, 2000).

[6] L. Carbone, 'Non-uniform lattices on uniform trees', Mem. Amer. Math. Soc. 152 (2001), 127.

[7] L. Carbone, 'The tree lattice existence theorems', C. R. Math. Acad. Sci. Paris 335 (2002), 223-228.

[8] L. Carbone and L. Ciobanu, 'Characterization of non-minimal tree actions', submitted.

[9] L. Carbone and D. Clark, 'Lattices on parabolic trees', Comm. Algebra . 30 (2002), 1853-1886.

[10] L. Carbone and G. Rosenberg, 'Lattices on non-uniform trees', Geom. Dedicata 98 (2003), 161-188.

[11] G. Rosenberg, Towers and covolumes of tree lattices, $\mathrm{PhD}$. Thesis (Columbia University, 2001).

[12] J.P. Serre, Trees, (Translated from the French by John Stilwell) (Springer-Verlag, Berlin, Heidelberg, 1980).

Department of Mathematics

Hill Center-Busch Campus Rutgers

The State University of New Jersey

110 Frelinghuysen Rd

Piscataway, NJ 08854-8019

United States of America

e-mail: carbonel@math.rutgers.edu 